\title{
On quasi-Armendariz properties on skew polynomial rings
}

\begin{abstract}
This paper deals with the quasi-Armendariz ring in general setting. We generalize the notions of quasi-Armendariz and Ǔ-skew Armendariz ring to quasi Ŭ-Armendariz and quasi Ŭ-skew Armendariz ring and investigate their properties. The notions of quasi ŬArmendariz and quasi Ŭ-skew Armendariz rings are useful in understanding the relationships between annihilators of rings $\mathrm{R}$ and $\mathrm{R}[\mathrm{x} ; \breve{\mathrm{U}}]$.
\end{abstract}

Keyword: Armendariz ring; Ŭ-quasi-Armendariz ring; Quasi Ŭ-skew Armendariz ring 